\title{
Face Recognition Based on Gabor Wavelet Transform and Modular 2DPCA
}

\author{
H. Yan, P. Wang, W.D. Chen , J. Liu \\ College of Computer Science \\ Chongqing University of Technology \\ Chongqing, China
}

\begin{abstract}
Since the dimension of face features which is presented by Gabor wavelet is too high, there has large computation if using the feature by Gabor wavelet transform for recognition directly. A novel idea based on Gabor wavelet transform and modular Two-principal component analysis for face recognition is proposed. Firstly, face image feature is acquired by Gabor Wavelet transforming. Secondly, its dimension is reduced and eigenvectors are extracted by the method of modular 2DPCA. Finally, fusion with nearest neighbor classifier and support vector machine (SVM) is adopted to sort and distinguish. Experimental results on ORL and YALE show that the performance of proposed method is superior to other methods, such as modular 2DPCA and combination of Gabor wavelet transform and 2DPCA.
\end{abstract}

Keywords-Modular two-dimensional principal component analysis(modular 2DPCA); gabor wavelet; face recognition; Support vector machine(SVM)

\section{INTRODUCTION}

Face recognition has an active development in the past few decades due to its potential applications in access control, intelligent surveillance, automated video surveillance, law enforcement and identity authentication. With the same as other biometric technologies, There have been a lot of methods proposed for overcoming the difficulty of face recognition. Such as linear[1-5]methods have been widely used in face recognition. The principal component analysis(PCA)[1-3]and the linear discriminant analysis(LDA)[4,5] are two typical examples of linear transform methods.

The Gabor wavelet representation facilitates recognition without correspondence because it captures the local structure corresponding to spatial frequency, spatial localization, and orientation selectivity. As a result, the Gabor wavelet representation of face images should be robust to variations due to illumination and facial expression changes[6]. Although Gabor wavelets have a unique advantage in face recognition, but the dimension of face features which is presented by Gabor wavelet is too high. there has large computation if using the feature by Gabor wavelet transform for recognition directly. Cao et al.[7]came up with a idea that used PCA to reduce the Gabor feature dimension. However, before PCA transform two-dimensional images need to be converted into a one- dimensional vector matrix, which easily lead to the curse of dimensionality. Ma el at[8] used 2DPCA to reduce Gabor feature. Unlike Eigen face in which the analysis and operation are based on $1 \mathrm{D}$ vector representation. 2DPCA is based on 2D matrix rather than $1 \mathrm{D}$ vector. Chen el at[9] proposed modular 2DPCA for face recognition. The method divides the face image into sub-images of the same dimension, which is helpful to highlight the local feature affected by posture, facial expression and so on. So it has been widely used in face recognition. The paper combines the Gabor wavelet transform and modular 2DPCA algorithm for face recognition and compares the recognition rate with PCA, LDA, Gabor wavelet transform combined with 2DPCA algorithm. The experimental results on ORL and YALE face database show that the algorithm has good recognition rate.

\section{FACE FEATURE EXTRACTION BASED ON GABOR WAVELETS}

Gabor wavelet transform has a good visual characteristics, and has a similar to mammalian visual system. It has the ability to time-frequency analysis combined with multi-scale and multi-directional characteristics. Gabor wavelet kernel function is defined as follows[10]:

$$
G_{u, v}(z)=\frac{\left\|k_{u, v}\right\|}{\partial^{2}} e^{\|-\| k_{u, v}\left\|^{2} \frac{\|z\|^{2}}{2 \partial^{2}}\right\|}\left[e^{i k_{u, v} z}-e^{\frac{-\partial^{2}}{2}}\right]
$$

Where $\quad k_{u, v}==\left\{\begin{array}{l}K_{v} \cos \phi_{u} \\ K_{v} \sin \phi_{u}\end{array}\right\}, K_{v}=\frac{k_{\max }}{f^{v}}, \phi_{u}=\frac{u \pi}{N}, z=(x, y), \mu$ and $\mathrm{v}$ defines the orientation and scale of the Gabor filters, $k_{\max }$ is the maximum frequency, and $f$ is the spacing factor between kernels in the frequency domain. $(x, y)$ represents the pixel coordinates. the spacing factor between kernels in the frequency domain. $\partial=2 \pi, k_{\max }=\frac{\pi}{2}, f=\sqrt{2}, \frac{k_{u, v}}{\partial^{2}}$ determines the size of the Gauss window function. Facial feature extraction, usually choose five dimensions, eight directions.

Gabor feature of Face image is obtained by $I(x, y)$ with a group of Gabor transform nuclear convolution. ts formula is as follows:

$$
H(u, v, x, y)=G_{u, v}(z) \otimes I(x, y)
$$


Where $H(u, v, x, y)$ is the face of Gabor image, the face feature information in different frequencies and the different directions can be obtained through the changes of $\mathrm{u}$ and $\mathrm{v}$ parameter. However, its dimension are very high, In order to reduce its dimension, this paper adopts module 2DPCA to reduce the dimension.

\section{MODULAR 2DPCA TRANSFORMATION}

The modular 2DPCA is the extension of the 2DPCA,module 2DPCA divides each image into sub-image for each sub-image with the process of the 2DPCA respectively .

Assuming that there are A different persons in face samples library, the $i_{\text {th }}$ person has $a$ training sample image matrixs: $I_{i 1}, I_{i 2}, I_{i 3}, \cdots, I_{i a},(i=1,2,3, \ldots, A)$. The size of training sample image $I_{i j}(i=1,2,3, \ldots, A, j=1,2,3, \ldots, a)$ is $M \times N$, The $M \times N$ Block matrix is expressed as follows:

$$
I_{i j}=\left\{\begin{array}{cccc}
I_{i j(11)} & I_{i j(12)} & \ldots & I_{i j(1 n)} \\
I_{i j(21)} & I_{i j(22)} & \ldots & I_{i j(2 n)} \\
\ldots & \ldots & \ldots & \ldots \\
I_{i j(m 1)} & I_{i j(m 2)} & \ldots & I_{i j(m n)}
\end{array}\right\}
$$

The size of each sub image is $p \times q(p=M / m, q=N / n)$. The mean of all the training sample :

$$
B=\frac{1}{M} \sum_{i}^{A} \sum_{j}^{a} \sum_{k}^{m} \sum_{l}^{n} I_{i j}(k l)
$$

$C=I_{i j}-B$ is the difference matrix between each sub image $I_{i j}$ and $B$ the average. The overall scattering matrix of the sub image:

$$
S=\frac{1}{M} \sum_{i}^{A} \sum_{j}^{a} \sum_{k}^{m} \sum_{l}^{n} C_{i j(k l)} \times C_{i j(k l)}{ }^{\prime}
$$

There $M=A \times a \times m \times n$ is the total number of sub image matrix of training samples. It is easy to prove that $S$ is the nonnegative definite. Similar to 2DPCA, Needing to find a set of optimal projection vectors $V_{1}, V_{2}, \ldots, V_{P}$. which meets the standarded orthogonality. Write it as $V=\left[V_{1}, V_{2}, \ldots, V_{P}\right], P \in R^{(p \times q) \times P}$, which is named by the optimal projection matrix. The training samples are projected to the optimal projection matrix, the formula is: $W_{i}=V^{T} \times\left(I_{i j}-B\right)$

\section{CLASSIFIER DESIGN}

Design a good classifier is also a very important process in face recognition. $\mathrm{K}$ - nearest neighbor method ( $\mathrm{K}$-nearest neighbor, KNN for short), and Support vector machine (SVM) are common classifiers[11-12]. KNN is a simple and nonparametric classification method in pattern recognition[11]. The main idea is comparing the Euclidean distance between test samples and all the training samples, the sample which is nearest to the test sample are of the same class. Supposing training sample $B_{i}$ belonged to the class $W_{c}$, a test sample $C_{j}$, The definition of the distance formula:

$$
d=\sum_{i=1}^{n}\left\|B_{i}-C_{j}\right\|_{2}
$$

$B_{i}, C_{j}$ are the characteristic matrixs. If the distance of $d\left(B_{1}, C_{1}\right)$ is minimal, $C_{j}$ belongs to $w_{c}$. SVM[12] is a supervised learning method, it has very good effects in dealing with high dimension data problems. Supposing a data set $\left\{\left(x_{1}, x_{2} \ldots x_{n}\right)\right\}, x \in R^{D}$, Class Label $y \in\{1,-1\}$, Our purpose is to find a hyper plane $\omega \cdot x+b=0$ making data separated. For the linear separable case, by solving a constrained extreme value problem, we can get the optimal classification plane. The optimal classification criterion function is:

$$
f(x)=\operatorname{sgn}\left(\sum_{i=1}^{n} a_{i} y_{i}\left(x_{i} \cdot x\right)+b\right)
$$

In this formula: $a_{i}, b$ is the optimal solution of the constrained extreme value problem. For the nonlinear separable, By introducing the kernel function $K\left(x_{i}, x_{j}\right)=\varphi\left(x_{i}\right) \cdot \varphi\left(x_{j}\right)$, The optimal classification criterion function (8) above turns into :

$$
f(x)=\operatorname{sgn}\left(\sum_{i=1}^{n} a_{i} y_{i} K\left(x_{i}, x\right)+b\right)
$$

This paper based on KNN and SVM, proposes a new classifier fusion method - KNN+SVM method.

The idea of the algorithm: Firstly, extract features from face image using Gabor wavelet and 2DPCA, Secondly use KNN to filter feature vector, Finally using the SVM to classify and recognize. The algorithm flow chart is as follows:

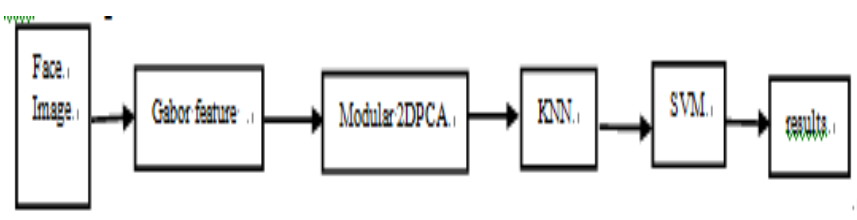

FIGURE I. KNN + SVM ALGORITHM FLOW CHART.

\section{EXPERIMENT AND ANALYSIS}

This experiment adopted the ORL and YALE face database respectively. ORL face database contains 40 people, everyone has 10 different image. Image contains certain illumination changes, expression change. The size of the image resolution is $112 \times 92$ pixels. YALE face database has 15 people, 165 
images. Where illumination, expression and posture change is big. This article on the ORL database selects five images as training sample, five as testing sample, also selects five as training sample, six image as testing sample on the YALE database. Each sample is normalized to $128 \times 128$. Figure 1 is one in ORL face database with 5 images and one in YALE with 5 images.

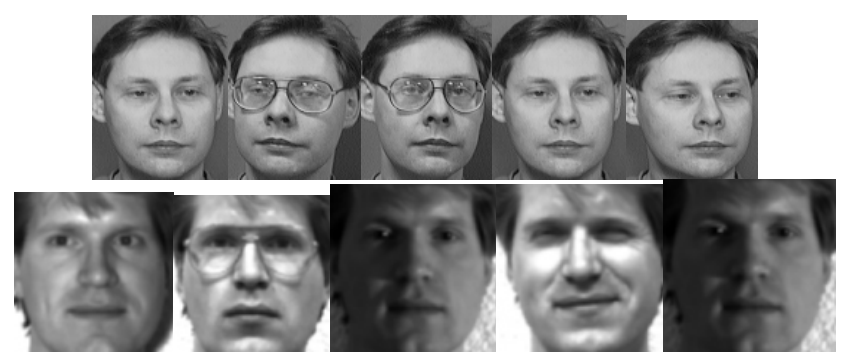

FIGURE II. THE FIRST ROW:5 IMAGES OF A MAN IN THE ORL DATABASE , THE SECOND ROW:5 IMAGES OF A MAN IN THE YALE DATABASE.

Figure 3 is the results of our methods and other methods. Each image is divided by four scheme as $2 \times 2,4 \times 2,4 \times 4,8 \times 4$, The size of each sub-image is $64 \times 64,32 \times 64,32 \times 32,16 \times 32$. So their feature vector can be easily obtained.

The recognition rate of blocking is better than not block from the Figure 2. This is because after the image is partitioned, the face local feature information can be highlighted, and these information for the illumination, expression has a certain robustness. But the number of partitioned is not the more the better, when by 16 blocks, recognition rate is the highest, then the recognition rate did not change obviously when it is by 32 blocks. So this paper selects the best way of blocks of $4 \times 4$.
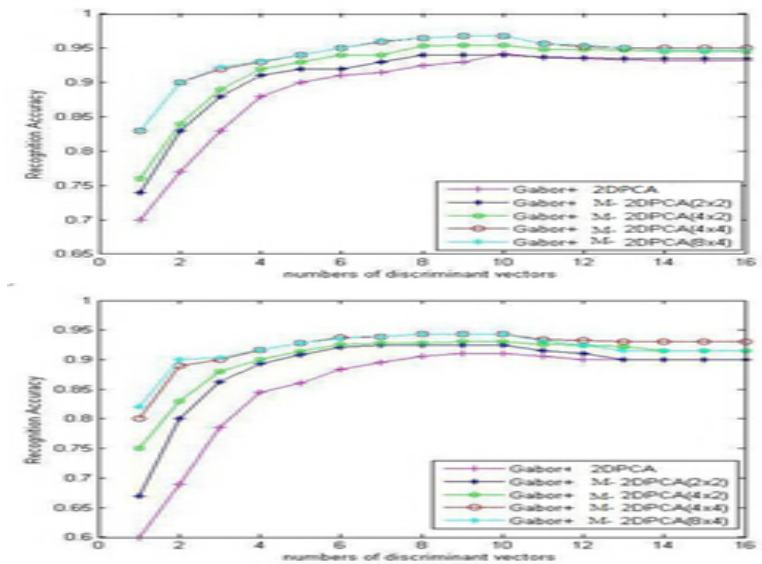

FIGURE III. THE RELATIONSHIP BETWEEN THE DIFFERENT BLOCK NUMBER RECOGNITION RATE AND THE FEATURE VECTORS IN THE 2 FACE DATABASES (\%).

Table 1 displays that combining with Gabor wavelet and modular 2DPCA algorithm has the highest recognition rate on ORL and YALE. It demonstrates that the algorithm in this paper can give a better description of the local face information, and the dimensions of the local information is low, Table 1 also shows that the YALE recognition rate is lower than that of the ORL. It mainly due to the YALE face database changed large in illumination, expression than ORL face database.

Since the KNN algorithm is a simple classification, SVM has a very good effect on class classification, some researchers combine these 2 methods to classify [13]. This methods of combination have been widely used to face recognition. This paper uses the combination The experimental results is table 2, it shows that: the highest recognition rate was $96.5 \%$ using $\mathrm{KNN}+\mathrm{SVM}$ in the ORL face database, 4.5\% higher than using KNN alone and $2.4 \%$ higher than that of SVM. The highest recognition rate of $\mathrm{KNN}+\mathrm{SVM}$ in the YALE database was 92.7\%, 4.3\% higher than KNN and 3.7\% higher than that of SVM. Therefore, KNN+SVM has a better classification ability than KNN or SVM individually.

TABLE I THE IDENTIFICATION RESULTS OF EACH ALGORITHM(\%).

\begin{tabular}{llccccc}
\hline \multicolumn{1}{c}{ method } & \multicolumn{2}{c}{ ORL database } & & \multicolumn{2}{c}{ YALE database } \\
& $\begin{array}{c}\text { optimal } \\
\text { projection } \\
\text { dimension }\end{array}$ & $\begin{array}{c}\text { best } \\
\text { feature } \\
\text { dimensio } \\
\text { n }\end{array}$ & $\begin{array}{c}\text { recognition } \\
\text { rate }\end{array}$ & $\begin{array}{c}\text { optimal } \\
\text { projection } \\
\text { dimension }\end{array}$ & $\begin{array}{c}\text { best } \\
\text { feature } \\
\text { dimension }\end{array}$ & $\begin{array}{c}\text { recognition } \\
\text { rate }\end{array}$ \\
\hline Gabor+modular2DPCA & 5 & $128 \times 5$ & 96.5 & 8 & $128 \times 8$ & 92.7 \\
Gabor+2DPCA & 10 & $128 \times 10$ & 94.5 & 12 & $128 \times 12$ & 91.5 \\
modular 2DPCA & 16 & $128 \times 16$ & 92.5 & 20 & $128 \times 20$ & 90 \\
PCA & 21 & 21 & 88 & 24 & 24 & 82 \\
Fisher & 18 & 18 & 92 & 24 & 24 & 89 \\
\hline
\end{tabular}

TABLE II COMPARISON OF DIFFERENT CLASSIFIER (\%).

\begin{tabular}{lll}
\multicolumn{1}{c}{ method } & \multicolumn{1}{c}{ ORL database } & \multicolumn{1}{c}{ YALE database } \\
\hline KNN & 92 & 88.4 \\
SVM & 94.1 & 89 \\
KNN+SVM & 96.5 & 92.7 \\
\hline
\end{tabular}




\section{CONCLUSION}

This algorithm makes full use of the property of the Gabor wavelet that it can effectively capture the face images of different spatial location, space, frequency and direction selectivity local structure characteristics, overcome the influence of illumination, scale, Angle , also modular 2DPCA can extract the feature whose dimensions are lower. Experimental results show that: the proposed algorithm has higher recognition rate than PCA, LDA and Gabor + 2DPCA.

\section{ACKNOWLEDGEMENTS}

This work is supported by National Natural Science Foundation of China (NO. 61173184) and the Graduate Innovation Foundation of Chongqing University of Technology (NO. YCX2013219).

\section{REFERENCES}

[1] Turk M, Pentland A. Eigenfaces for recognition [J]. Journal of Cognitive Neuroscience, 1991, 3(1): 71-86.

[2] Xiong Chengyi, Li Danting, Da Bangyou .Face recognition based on LBP and PCA feature extraction [J]. Journal of South-Central University for Nationalities,2011,(2):75-79.

[3] Yin Yong, Wen Juan. Face recognition based on modular PCA and SVD[J]. Journal of Chongqing University.2012,08:134-138.

[4] Zhang Jian, Xiao di. Face recognition method based on multi-scale adaptive LDA[J]. Computer Engineering and Design, 2012, 33(1): $332-335$.

[5] LU Guifu, ZOU Jian, WANG Yong. Incremental complete LDA for face recognition[J]. Pattern Recognition, 2012, 45(7):2510-2512

[6] C. J. Liu et al.Gabor-Based Kernel PCA with Fractional Power Polynomial Models for Face Recognition[J], IEEE Trans. On PAMI, Vol. 26, No. 5,2004: 572-581.

[7] C. Liu, H Wechsler. Independent component analysis of Gabor features for face recognition[J], IEEE Trans. Neural Networks, Vol. 14, No. 4, 2003:919-928.

[8] Ma xiao yan et al.Face recognition based on Gabor wavelet and 2DPCA Computer Engineering and Applications[J].2006.10:55-57.

[9] CHEN Fu-bing, CHEN Xiu-hong, ZHANG Sheng-liang et al. A Human Face Recognition Method Based on Modular 2DPCA.Joumal of Image and Graphics[J].2006,11(4):580-585.

[10] T. S. Lee, Image Representation Using 2D Gabor Wavelets, IEEE Trans. On PAMI, Vol. 18, No. 10, October 1996,pp. 959-971.

[11] Cover T, Hart P. Nearest neighbor pattern classification[J].Information Theory, 1967, 13(1): 21-27.

[12] Schwenker F.Hierarchical Support Vector Machines for Multi-class Pattern Recognition[C]/Proceedings of the 4thIntenrational Conference on Knowledge-based Intelligent Engineering Systems\& Allied Technologies. UK : University of Brighton, 2000: 561-565.

[13] CHEN Zhen zhou, LI Lei, YAO Zhengan. Feature-weighted K-Nearest neighbor algorithm with SVM[J]. Acta Scientiarum Naturalium Universitatis Sunyatseni, 2005, 44(1): 17-20. 